\title{
A new cure for hypertension - Renal Denervation
}

\author{
Niamh Goulding
}

Department of Physiology, UCC

The kidney presents in the highest degree the phenomenon of sensibility, the power of reacting to various stimuli in a direction which is appropriate for the survival of the organism; a power adaptation which almost gives one the idea that its component parts must be endowed with intelligence. (Ernest Starling 1909)

\section{Introduction}

Hypertension, or high blood pressure, is estimated to effect one billion people worldwide. It contributes to an estimated 7.1 million deaths annually. Patients with hypertension are at a greater risk to stroke, coronary artery disease, heart failure, vascular disease, and chronic renal failure. Approximately $65 \%$ of hypertensive patients are either untreated and/or uncontrolled. Out of these, 10\% have a particular type of hypertension called resistant hypertension. This is a form of hypertension that is drug-resistant. This means it is not controlled by antihypertensive agents/drugs. This particular type of hypertension is commonly seen in patients with renal failure (RF). It has been shown recently that this association of hypertension with renal failure may be a result of inappropriate sensory nerve activity arising from the failing kidneys.

Vito Campese was among the first to suggest that the hypertension associated with renal failure (RF) could be attributed to the inappropriate stimulation of the sensory nerves in the kidney itself. This hypothesis was built on by studies by Hausberg et al. They showed that in patients with end stage renal failure there was elevated nerve activity in the patients who, during kidney transplantation, did not have the diseased kidney removed (see figure 1).

This indicated a key role for renal sensory nerves within the diseased kidney in determining nerve output which can be significantly over stimulated in RF. This nerve activity has a knock-on effect of increasing blood pressure and thereby leading to a hypertensive state. A new potential therapeutic approach for controlling such hypertension is to disrupt or denervate the nerve pathways between the brain and the kidney. 


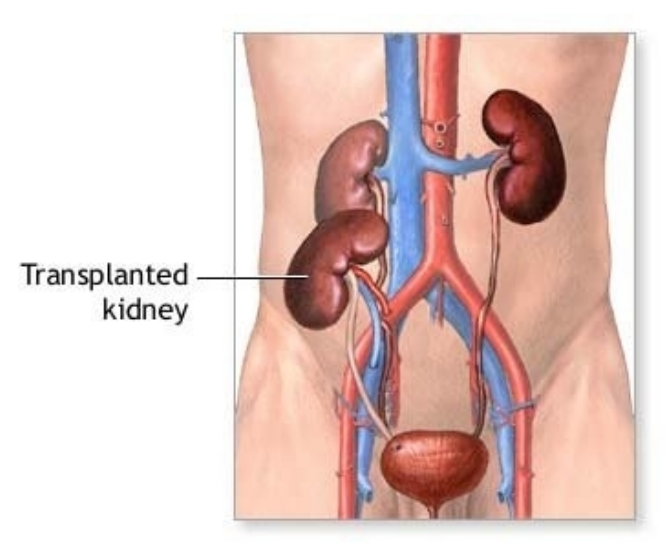

Figure 1: Kidney Transplantation

\section{My doctoral research}

My research was undertaken to further demonstrate that during renal failure there is an inappropriate increase in nerve activity arising from the diseased kidney and that this leads to a disregulation of normal cardiovascular function and reflexes.

The kidney is essential in controlling blood pressure. It does this by controlling fluid balance or blood volume. By controlling body fluid, the kidneys can control our blood pressure (BP). When BP is increased, our kidneys excrete fluid to lower BP. Conversely, when BP decreases, our kidneys retain fluid driving BP up. Figure 2 shows how as body fluid increases, this results in more fluid in the blood thus increasing blood volume or the amount of blood in our bodies. This directly increases BP as a result. Therefore by controlling fluid loss and fluid retention our kidneys regulate BP.

The sympathetic nervous system, a division of the body's involuntary nervous system is an important contributor to the control of arterial pressure under everyday perturbations in blood pressure. It does this under varying conditions by altering cardiac output (the amount of blood the heart pumps), peripheral resistance (diameter of blood vessels) and also renal function. Renal control of arterial pressure is regarded as being the major determinant of long-term arterial pressure because of its crucial role in maintaining extracellular fluid volume.

The kidneys are vitally important in altering arterial pressure by either increasing or decreasing fluid reabsorption and secretion in response to varying blood pressures. Activa-

Figure 2: How the body fluid directly affects blood pressure 


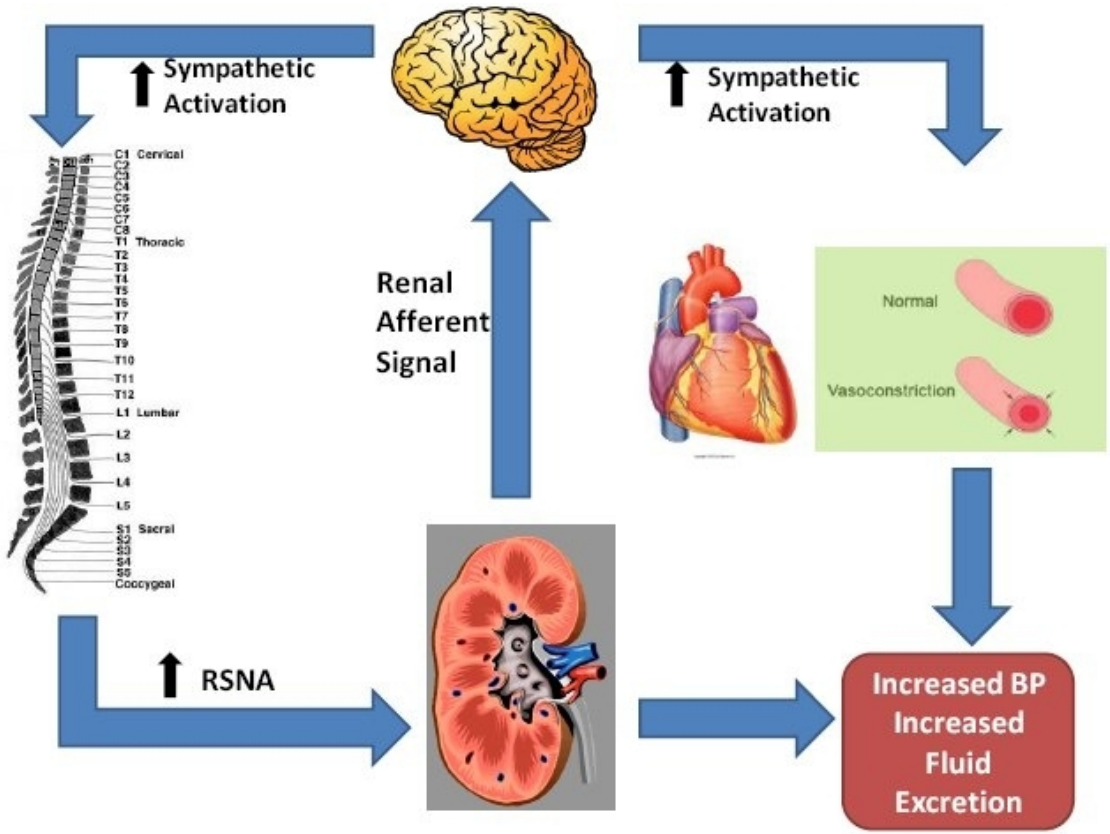

Figure 3: Schematic of the interaction between the kidney, brain and cardiovascular system

tion or inhibition of what is called renal sympathetic nerve activity (RSNA) is critical in this role (see figure 3). In a healthy kidney RSNA when activated will cause a decrease in filtration rate within the kidneys, thereby decreasing water loss and as a result increasing BP. In situations where BP has decreased, the brain stimulates RSNA. Conversely, the brain inhibits RSNA when BP has increased; this causes fluid loss and results in a reduction in BP. It is this reflex, known as the baroreflex, that I examined during my research.

Baroreceptors are the pressure sensors in our bodies. There are two types; high and low pressure baroreceptors. High pressure baroreceptors are activated by sudden changes in $\mathrm{BP}$, whereas low pressure baroreceptors are active during slower, more gradual perturbations in BP.

My study investigated how the reflex regulation of renal sympathetic nerve activity was altered in a rodent model of renal failure.

\section{Methodology and results}

The high pressure baroreceptors were examined by causing sudden increases and decreases in BP. From recorded data, a baroreflex gain curve was generated. This curve (shown in figure 4) shows how as BP increases RSNA decreases and as BP decreases RSNA increases in a normal healthy control rodent model. So a normal baroreflex gain curve should be sigmoidal in shape.

However, as can be seen in Figure 4, this reflex is completely blunted in the RF model. This indicated that the RF model has a loss of its reflex control of BP through RSNA. In other words, the RF model could neither increase nor decrease the RSNA, suggesting that 


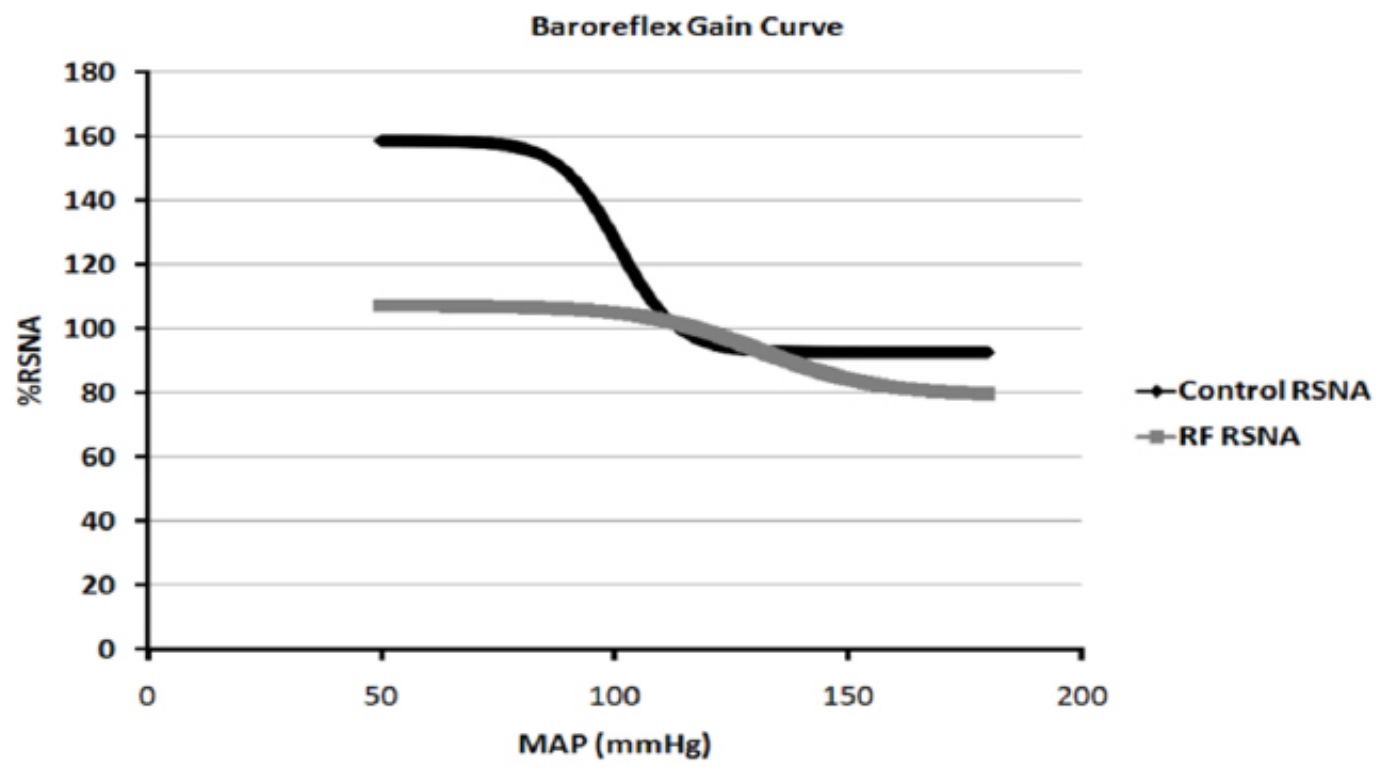

Figure 4: Baroreflex Gain Curve- Control Vs RF

RSNA was at a constant high level.

The low pressure baroreceptors were challenged by infusing saline over a 30min period, also known as a volume expansion. Infusing saline at an increasing but steady rate increases BP gradually. As BP increases over this 30 minute period RSNA should decrease to combat the effects. This is exactly what happens in the controls. Again, as can be seen in figure 5 this reflex is blunted in the RF model.

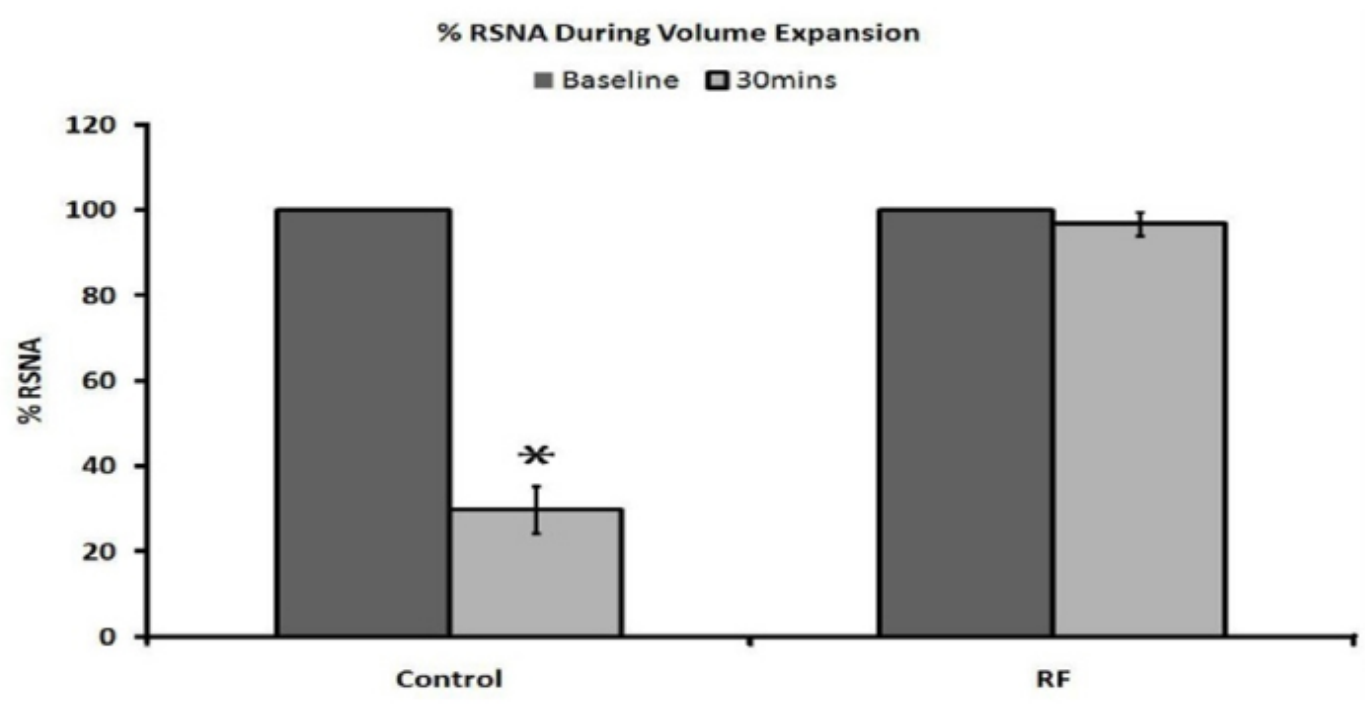

Figure 5: Graphs comparing reflex decrease in RSNA in control Vs RF rats 


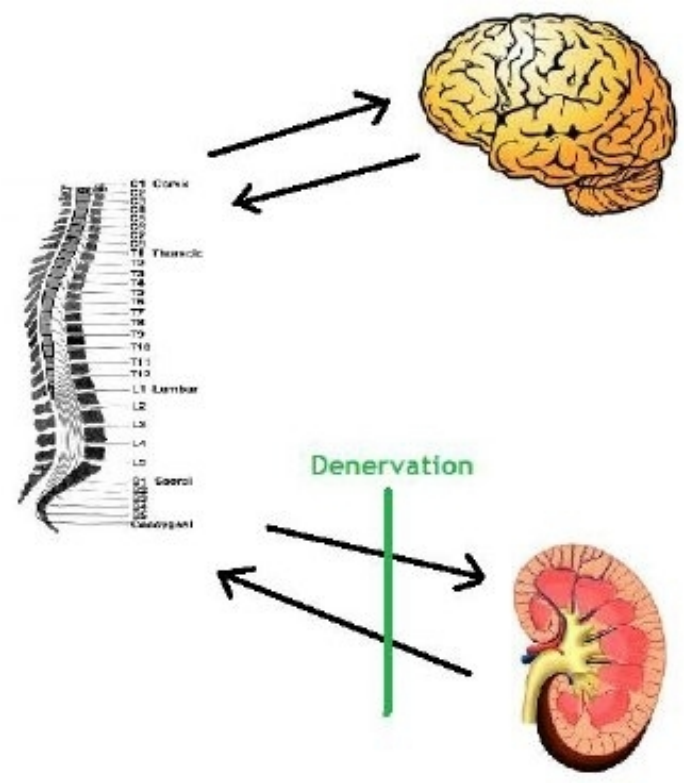

Figure 6: Diagram of renal denervation

Both baroreflexes were significantly different between control and RF models. Controls showed that during periods of increased BP, RSNA was decreased. However this was not true of the RF models. In both experiments RF models were not able to control BP via RSNA and the kidneys.

\section{Discussion}

Sympathetic hyperactivity plays a vital role in the hypertension associated with renal failure. As previously discussed hypertension is common in renal failure. Signals arising in the failing kidneys may mediate sympathetic activation with a simultaneous increase in renal afferent nerve activation.

A new therapeutic approach in treating patients with resistant hypertension, and that has shown significant success, is renal denervation (see figure 6). This involves disrupting the neural pathway between the brain and the kidneys, thereby stopping any inappropriate sensory information being sent to the brain which would then cause changes in RSNA. Doing this would not only stop the inappropriate sensory signal from the kidney getting to the brain, but also stop the brain nerve signals getting to the kidneys.

The next part of my research is to surgically denervate kidneys in the RF rodent models and to again look at their reflex control of BP to determine what effect denervation will have.

Thanks to my supervisor Professor Edward Johns and colleagues. 


\section{References}

\section{Books of interest}

Campese, Vito Renal Afferent Denervation Prevents Hypertension in Rats with Chronic Renal Failure, Hypertension, 1995

Hausberg, Martin, Sympathetic Nerve Activity in End-Stage Renal Disease, Circulation, 2002 